\title{
Correlated Local Atomic Displacements: The Microscopic Origins For Macroscopic Phenomena.
}

Frank Bridges ${ }^{1}$, Daliang Cao ${ }^{1}$, and Corwin H. Booth ${ }^{2}$

${ }^{1}$ Department of Physics, University of California, Santa Cruz, CA 95064

${ }^{2}$ Lawrence Berkeley National Laboratory, Berkeley, CA 94720

\begin{abstract}
In many systems for which there are several atoms in the unit cell, the displacements of the atoms may be locally correlated even though there is no long range coherence. Such displacements can play an important role in determining various macroscopic properties. We consider several examples to demonstrate this phenomena - the local distortions in the colossal magneto-resistive (CMR) and charge-ordered manganites, magnetic field induced distortions in CMR materials that are connected to macroscopic magnetostriction, and correlated displacements of tetrahedral units within the negative thermal expansion material $\mathrm{ZrW}_{2} \mathrm{O}_{8}$. The distortions in the $\mathrm{CMR}$ materials observed using XAFS change rapidly just below $\mathrm{T}_{c}$ and are attributed to the formation of polarons as the temperature is increased through $\mathrm{T}_{c}$. In the ferromagnetic state, the lattice is more ordered for CMR systems; consequently applying a magnetic field for $\mathrm{T} \sim \mathrm{T}_{c}$ should decrease the local distortions. Such an effect has been observed and is a much larger effect than the measured macroscopic magnetostriction. Finally, in $\mathrm{ZrW}_{2} \mathrm{O}_{8}$ the tetrahedral and octahedral units are found to be very rigid as expected. More surprising is that the width of the pair-distance distribution for the $\mathrm{W}-\mathrm{Zr}$ atom-pair is also quite small, indicating that the $\mathrm{W}-\mathrm{Zr}$ linkage is stiff. In contrast, for the $\mathrm{W}-\mathrm{W}$ pair, the distribution width grows rapidly with $\mathrm{T}$, indicating correlated displacements of two $\mathrm{WO}_{4}$ tetrahedral units.
\end{abstract}

\section{INTRODUCTION}

Correlations of the motions and displacements of the constituent atoms in a crystal occur for all materials, but their relevance in determining some of the macroscopic properties of a material have only recently begun to be understood. For simple materials such as the alkali halide salts, the dominant vibrational modes at low temperatures are the acoustic phonons. For these modes, particularly for long wavelength modes, the displacements of the neighboring atoms are highly positively correlated - that is at any instant in time, these atoms are moving in the same direction[1]. At higher temperatures, the optical phonons become excited; for these modes the motions of the atoms are negatively correlated[2, 3], particularly for short wavelength modes near the Brillouin zone boundary. In this case, the neighboring atoms move towards or away from each other and if charged (ionic), couple strongly to electromagnetic radiation (i.e. visible and infrared light). Eventually at high enough temperatures that a large number of short wavelength modes of various types are excited, the net motions of the neighboring atoms should become essentially uncorrelated. For simple systems, apart from the coupling to optical radiation, the correlated motions of the atoms do not appear to play a major role in most macroscopic properties.

However for other systems, often those with larger more complex unit cells, the situation is changed. Additional correlations may be present, and in unit cells containing many atoms, there 
are a very large number of optical modes[4] which can easily dominate the acoustic vibrations. One example of another type of atomic correlation is the set of displacements that follow a hopping polaron - typically an elongation of the bonds in two directions and a shortening of the bonds in the other four directions for perovskite systems. These local distortions are electronically driven and should not be confused with the normal modes of the system (solutions of the dynamical matrix) that produce the phonons. However there may be a small electron-phonon coupling between the polaron and the phonons. When the optical phonon modes dominate the system, some of them may be soft modes (low frequency)[4] and in this case some of the displacements of atoms in the unit cell can be negatively correlated even at low temperatures. We consider both situations here.

One can experimentally probe the degree of correlation of the displacements of some pair of atoms by combining results from XAFS (X-ray Absorption Fine Structure) with those of diffraction[2]. Consider two atoms in the crystal, A and B, and let their local displacements along a line between the atoms be $\delta x_{A}$ and $\delta x_{B}$ respectively. In XAFS one obtains the width of the pair distribution function, $\sigma_{A B}$, by fitting the data to standards. The variance $\sigma_{A B}^{2}$, is the average of $\left(\delta x_{A}-\delta x_{B}\right)^{2}$ over the lattice, given by:

$$
\sigma_{A B}^{2}=<\left(\delta x_{A}-\delta x_{B}\right)^{2}>=<\delta x_{A}^{2}>+<\delta x_{B}^{2}>-2<\delta x_{A} \delta x_{B}>,
$$

where $<>$ denotes a spatial average. In diffraction, the thermal parameters, $\mathrm{U}_{A}^{2}, \mathrm{U}_{B}^{2}$, are the average of the squared displacements about a lattice site - for example for atom $\mathrm{A}, \mathrm{U}_{A}^{2} \sim$ $\left.<\left(\delta x_{A}\right)^{2}\right)>$. There is actually a slight difference here in the definition of $\delta x_{A}$ - for XAFS it is measured from each atomic site but in diffraction it is the displacement from a uniformly spaced set of mathematical points. When the sample is homogeneous, these are the same but for some inhomogeneous systems the distinction may be important, as then $\mathrm{U}^{2}$ and $\sigma_{A B}^{2}$ have different static contributions. In Eqn. 1 the important quantity for correlation effects is the last term $-2<\delta x_{A} \delta x_{B}>$. If the motions of the atoms $\mathrm{A}$ and $\mathrm{B}$ are independent (i.e. uncorrelated) then this term is zero; if the displacements are positively correlated this term will make $\sigma_{A B}^{2}$ small while if the correlations are negative, $\sigma_{A B}^{2}$ is greater than the sum $\mathrm{U}_{A}^{2}+\mathrm{U}_{B}^{2}$. We have defined a correlation parameter $\phi$ by setting $[2,3]$

$$
<\delta x_{A} \delta x_{B}>=\phi U_{A} U_{B}
$$

By comparing the values of $\sigma_{A B}^{2}$ with $\mathrm{U}_{A}^{2}+\mathrm{U}_{B}^{2}$ one can determine $\phi$.

If one is interested in the relative correlations of the displacements which are generated by different phonon modes being excited, (i.e. an acoustic and optic mode) then $\delta x_{A}$ and $\delta x_{B}$ must each be written as a superposition of the displacements for each of the excited modes $\mathrm{m}_{i}$;

$$
\delta x_{A}=\delta x_{A_{m_{1}}}+\delta x_{A_{m_{2}}} \cdots
$$

To carry out the calculation one needs to know how the motions of the atoms for each mode are correlated. Assuming the displacements for a given mode are correlated but those of the two modes are not, then

$$
\sigma_{A B}^{2}=\sigma_{A B_{m_{1}}}^{2}+\sigma_{A B_{m_{2}}}^{2}
$$



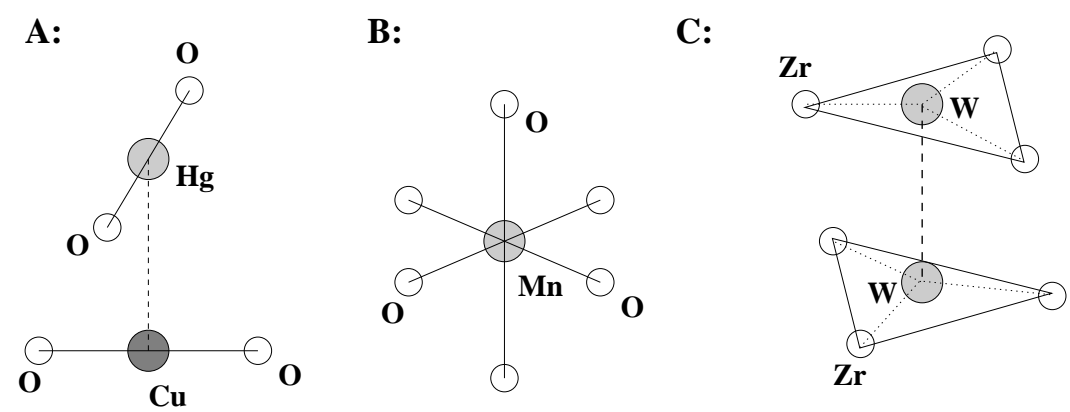

Figure 1: Units within crystal structures that have correlated displacements. A: The $\mathrm{HgO}_{2}$ dumbbell in the $\mathrm{HTC}$ superconductor $\mathrm{HgBa}_{2} \mathrm{CuO}_{4+\delta}$ (the $\mathrm{O}$ neighbors about $\mathrm{Hg}$ are labelled $\mathrm{O}(2)$ ) which moves as a rigid unit. Normally this unit is aligned along the vertical axis (c-axis), but the $\mathrm{Cu}-\mathrm{O}(2)$ linkage is very disordered, so some rotation (exaggerated here) may take place. B: The (correlated) displacements of $\mathrm{O}$ around $\mathrm{Mn}$ in a JT configuration. As the polaron hops, this distortion follows the charge. $\mathrm{C}$ : The units within $\mathrm{ZrW}_{2} \mathrm{O}_{8}$ that are important for negative thermal expansion. There is an octahedron of $\mathrm{O}$ about each $\mathrm{Zr}$ and a tetrahedron of $\mathrm{O}$ about each $\mathrm{W}$ atom. The two $\mathrm{W}$ atoms are $\mathrm{W}_{1}$ and $\mathrm{W}_{2}$ and the (111) axis is vertical in this figure.

where

$$
\sigma_{A B_{m_{1}}}^{2}=<\left(\delta x_{A_{m_{1}}}-\delta x_{B_{m_{1}}}\right)^{2}>
$$

Some examples of correlated displacements are shown in Fig. 1. On the left side is a part of the structure for the single $\mathrm{CuO}_{2}$ layer $\mathrm{Hg}$ superconductor, $\mathrm{HgBa}_{2} \mathrm{CuO}_{4+\delta}$. The $\mathrm{HgO}_{2}$ dumbbell is situated between two $\mathrm{CuO}_{2}$ layers and moves as a single unit - the motions of the $\mathrm{Hg}$ and the two $\mathrm{O}(2)$ atoms are very highly correlated[2]. In contrast, the displacements of the $\mathrm{O}(2)$ atoms (and hence the $\mathrm{HgO}_{2}$ unit) relative to $\mathrm{Cu}$ are negatively correlated. Whether this correlated motion is relevant to superconductivity is not clear.

The unit that represents the lattice displacements surrounding a polaron at some instant in time is shown in the middle of Fig. 1. There is a $\mathrm{Mn}$ atom in the center and six $\mathrm{O}$ atoms around it in a distorted octahedra - 2 long and 4 short $\mathrm{Mn}-\mathrm{O}$ bonds. Within the polaron model the local environment about a given $\mathrm{Mn}$ atom fluctuates between this unit and a nearly undistorted octahedron of $\mathrm{O}$ atoms as the polaron hops from site to site. In contrast to the $\mathrm{HgO}_{2}$ dumbbell considered above, these correlated displacements strongly influence the observed macroscopic properties - they enhance the metal/insulator transition and produce unusual thermal expansion and magnetostrictive effects.

Finally on the right side of Fig. 1 we show a combination of larger units $-\mathrm{WO}_{4}$ tetrahedra located within triangles of $\mathrm{ZrO}_{6}$ octahedra (this is just a part of the unit cell). For $\mathrm{ZrW}_{2} \mathrm{O}_{8}$, we propose that it is the the correlated motions of these units that produces the observed negative thermal expansion. We consider the latter two examples in more detail in the following sections.

\section{POLARON SYSTEMS}

Polarons are charge carriers that carry a local distortion of the lattice with them as they move throughout the crystal. The understanding of these quasiparticles and their local distortions 


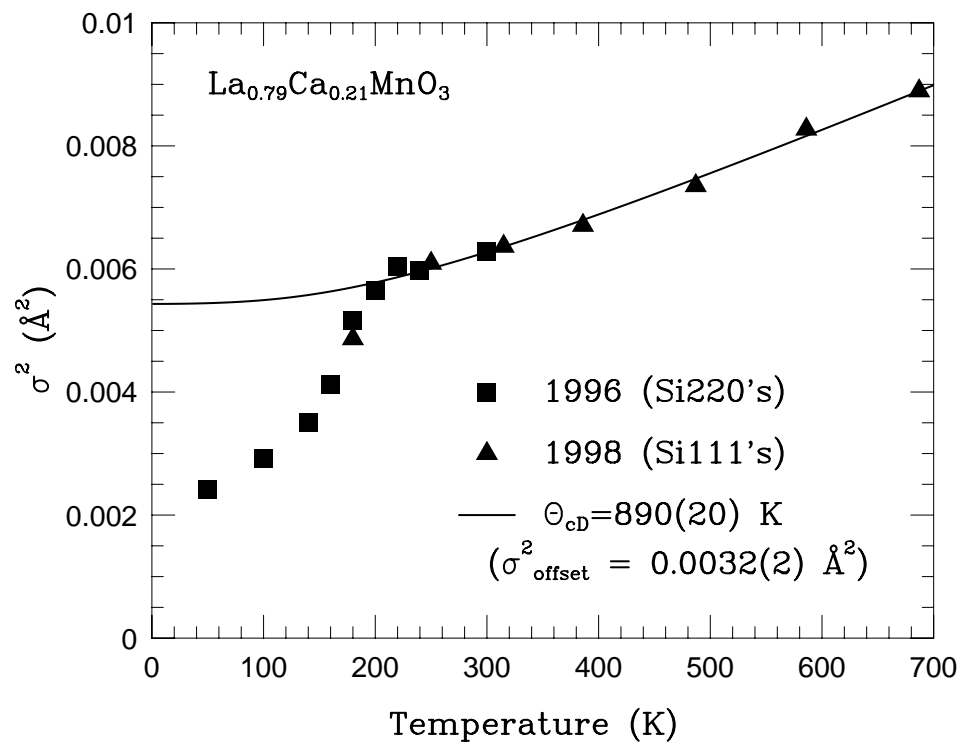

Figure 2: A plot of $\sigma_{M n O}^{2}$ for the $21 \%$ Ca sample, as a function of temperature. The data were collected in two different experimental runs on two Si monochromators: (220)'s - T up to 300K, and (111)'s - T from $180 \mathrm{~K}$ to $690 \mathrm{~K}$. The two runs were normalized for temperatures between 200 and $300 \mathrm{~K}$, to correct for the small differences obtained using two different monochromators (a multiplicative factor of 1.025 was included for the high temperature data). The solid line is a fit of the high temperature data to the correlated Debye model using $\sigma_{\text {off set }}^{2}=.0032 \AA$ as the effective static contribution.

have greatly increased over the last decade as a result of the intense study of the manganite systems which exhibit colossal magnetoresistance (CMR) (for the quasicubic manganites (perovskites) $\mathrm{La}_{1-x} \mathrm{Ca}_{x} \mathrm{MnO}_{3}, \mathrm{CMR}$ is observed for $0.2<\mathrm{x}<0.5$ ). At low temperatures, these materials are ferromagnetic metals and the lattice is highly ordered (the Mn-O pair distribution function is narrow). Above $\mathrm{T}_{c}$, conduction takes place via polaron hopping and the samples have a high resistance (semiconducting-like behavior) and are paramagnetic. The coupling between transport and magnetism is generally modeled using the double exchange (DE) model[5, 6, 7].

When polarons form there is a large additional increase in the local distortions about a lattice site, generally referred to as a Jahn-Teller (JT) distortion[3, 8, 9, 10, 11, 12]. This distortion enhances the increase in resistivity as the sample becomes paramagnetic $[13,14,15]$. Thus there is a strong coupling between the spins, the charge, and the lattice in these materials. In XAFS this results in an increase in the broadening $\sigma_{M n O}$, of the $\mathrm{Mn}-\mathrm{O}$ pair distribution function as $\mathrm{T}$ increases through $\mathrm{T}_{c}$.

In Fig. 2 we plot $\sigma_{M n O}^{2}$ as a function of temperature up to $700 \mathrm{~K}$ for a $21 \%$ Ca substituted sample. If we ignore the low $\mathrm{T}$ data the slow increase of $\sigma_{M n O}^{2}$ at high temperatures indicates a large Debye (or Einstein) temperature of order 900K. This means the average Mn-O bonds (springs) are quite stiff. However the large drop in $\sigma_{M n O}^{2}$ below $\mathrm{T}_{c}=190 \mathrm{~K}$ indicates the situation is more complex; the large change in $\sigma_{M n O}^{2}$ from $50 \mathrm{~K}$ to $\mathrm{T}_{c}$, is the signature for the formation of polarons. To separate these contributions, one must consider the correlations in the local distortions. We assume that the local displacements produced by phonons, polarons, and static 
distortions (caused by defects) are uncorrelated. Then $\sigma_{M n O}^{2}$ will be the sum of several terms:

$$
\sigma_{M n O}^{2}=\sigma_{\text {static }}^{2}+\sigma_{\text {polarons }}^{2}+\sigma_{\text {phonons }}^{2} .
$$

The XAFS data for the CMR samples are easily decomposed in this way; the static contribution is very small while the polaron contribution dominates until quite high temperatures are reached. The large increase in the width of the pair distribution function near $\mathrm{T}_{c}$ is also connected with the unusual thermal expansion observed, but the local structure changes are roughly an order of magnitude larger. This is expected - if an $\mathrm{O}$ atom moves towards one Mn atom and away from another, then the $\mathrm{Mn}-\mathrm{O}$ distribution would broaden even if the lattice has not expanded. However, the longer Mn-O bond is expected to lengthen more than the short bond contracts - this leads to the net expansion observed in diffraction. The resulting distribution of long and short bonds throughout the crystal is the microscopic basis for the increased thermal expansion observed just below $\mathrm{T}_{c}$. Note this contribution to the thermal expansion is electronically driven - it is not an anharmonic phonon effect.

\section{MAGNETOSTRICTION}

In the CMR materials the distortions of the Mn-O bonds are observed to decrease as the temperature is lowered below $\mathrm{T}_{c}$; at the same time, the sample magnetization increases. We have shown previously that for $\mathrm{T}<\mathrm{T}_{c}$, the decrease in the polaron contribution to $\sigma_{M n O}^{2}, \Delta \sigma_{\text {polaron }}^{2}$, is exponentially related to the sample magnetization, $\mathrm{M}\left(\Delta \sigma_{\text {polaron }}^{2}=\mathrm{AM} / \mathrm{M}_{o}+\mathrm{B}\right.$, where $\mathrm{A}$ and $\mathrm{B}$ are constants and $\mathrm{M}_{o}$ is the saturation value of $\mathrm{M}$ at low $\mathrm{T}$ ). This is attributed to a decrease in the number of Mn sites that have a JT distortion in regions where the sample becomes magnetic[9]. Consequently, it suggests that, at a fixed temperature, the sample should become more ordered when a magnetic field is applied. This is in fact the case - a small decrease in $\sigma_{M n O}^{2}$ is observed in a 1 T field[16]; however, due to the small magnetic field used, the effect was difficult to measure. To improve our ability to probe this effect, we have developed a magnetic field modulation technique[17] which determines the magnetic-field induced change in the XAFS when a magnetic field is pulsed on and off (on and off times are a few seconds). This generates a difference file that is the magnetic-field induced change in the XAFS. If this change is mainly the result of a change in $\sigma_{i}$ (for shell $\mathrm{i}$ ) then we can estimate the difference function using a Taylor expansion of the $\mathrm{XAFS}$ function $\mathrm{k} \chi \cdot \mathrm{k} \chi$ is defined by

$$
k \chi=\sum_{i} \frac{N_{i} S_{0}^{2} e^{-2 k^{2} \sigma_{i}^{2}}}{R_{i}^{2}} \sin \left(2 k R_{i}+\Phi_{i}\right)=\sum_{i} k \chi_{i} .
$$

where $\mathrm{N}_{i}$ is the number of atoms in shell $i, \mathrm{~S}_{0}^{2}$ is an amplitude reduction factor, which corrects for many-body effects, $\mathrm{R}_{i}$ is the average distance to atoms in shell $i, \mathrm{k}$ is the photoelectron wave number, $\sigma_{i}$ is the pair distribution width, and $\Phi_{i}$ is the total phase shift in the central and backscattering process. The Fourier Transform (FT) of this function yields peaks in r-space corresponding to the various shells of neighbors.

Differentiating this expression with respect to $\sigma_{i}$ we can obtain the change in $\mathrm{k} \chi$ generated 

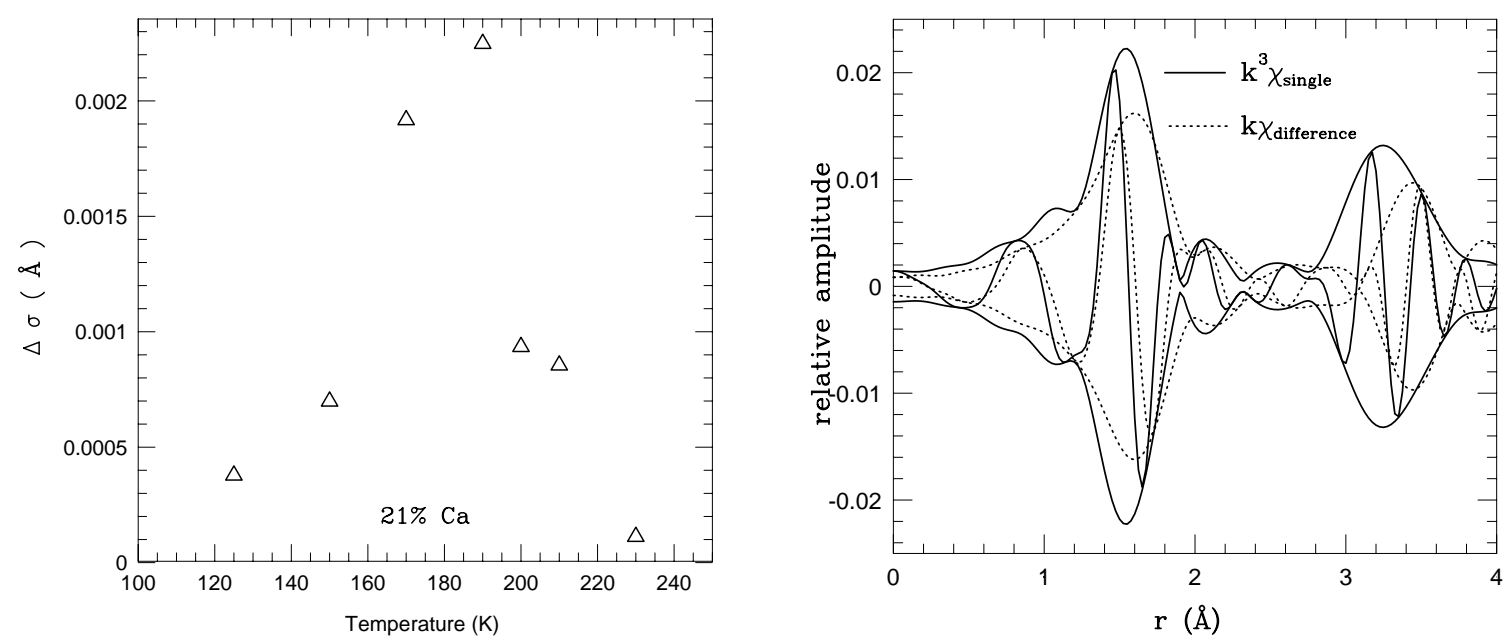

Figure 3: Left: The change in $\sigma$ induced by a 1T magnetic field for the Mn-O bond as a function of temperature. right: A comparison of the FT of the difference data with a $\mathrm{k}^{3} \chi$ function at zero field - the latter is reduced by a factor of 625 for comparison.

by a magnetic-field-induced change in $\sigma$.

$$
k\left(\Delta \chi_{i}\right)=\left(-4 k^{2} \sigma_{i}\right) \frac{N_{i} S_{0}^{2} e^{-2 k^{2} \sigma_{i}^{2}}}{R_{i}^{2}} \sin \left(2 k R_{i}+\Phi_{i}\right) \Delta \sigma_{i}=-4 \sigma_{i} \Delta \sigma_{i}\left(k^{3} \chi_{i}\right) .
$$

Thus the difference file obtained experimentally, $\mathrm{k} \chi_{\text {diff }}$, should be a sum of terms $A_{i} k^{3} \chi$. The amplitude $A_{i}=-4 \sigma_{i} \Delta \sigma_{i}$, is a direct measure of the small change in $\Delta \sigma_{i}$. In Fig. 3a we plot $\Delta \sigma_{M n O}$, obtained from the amplitude $\mathrm{A}_{1}$, as a function of $\mathrm{T}$. It shows a clear peak near $\mathrm{T}_{c}=190 \mathrm{~K}$, with a maximum change of $\sim 0.0023 \AA$. The application of a magnetic field has reduced the disorder - we interpret this as a reduction in the number of JT distorted sites in the lattice. However for a $1 \mathrm{~T}$ field, only a small fraction of the sites are affected.

In Fig. $3 b$ we compare the difference data with a $\mathrm{k}^{3} \chi$ trace, with the latter reduced in amplitude by a factor of 625 to be on the same scale as the difference data. The Mn-O and Mn-Mn peaks are clearly observed in the difference data at $\sim 1.6$ and $3.4 \AA$ respectively. However, the Mn-La peak which occurs near 3.1-2 $\AA$ is not observed in the difference data changes in this peak are at least a factor of 10 smaller.

The ratio of the change in broadening to the bond length, $\Delta \sigma_{M n O} / r_{M n O} \sim 10^{-3}$, is very large compared to macroscopic magnetostriction measurements which measure the average relative change of the lattice constant $\delta r / r$ when a field is applied; magnetostriction results are typical at least an order of magnitude smaller. The relative change of the pair-distribution width for $\mathrm{Mn}-\mathrm{Mn}$ in the difference data is comparable to that for $\mathrm{Mn}-\mathrm{O}$.

This difference in magnitudes of the local structure distortions and the macroscopic elongations observed in thermal expansion and magnetostriction arise from the difference in the quantity being measured. Changes in $\sigma^{2}$ are produced when the bond lengths locally are lengthened or shortened, whereas the net change in $\delta r$ is averaged over many unit cells. Consider a chain of $\mathrm{Mn}-\mathrm{O}-\mathrm{Mn}$ atoms in which the $\mathrm{Mn}-\mathrm{O}$ bonds are randomly shortened or lengthened corresponding to different local orientations of the JT distortion. This produces a distribution of 


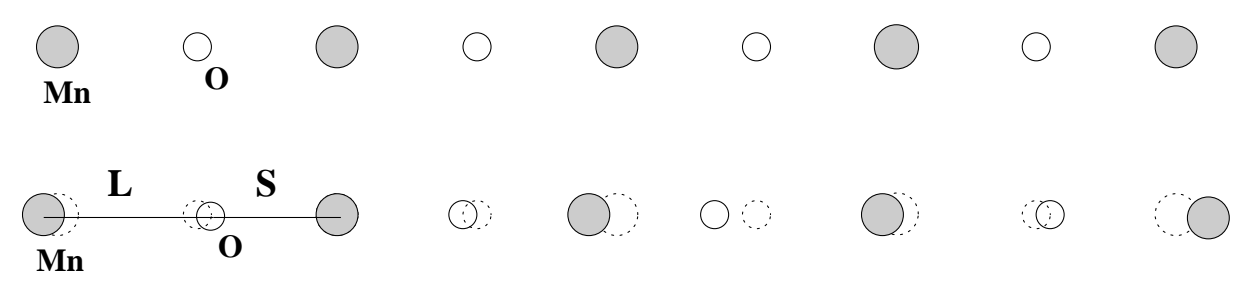

Figure 4: A schematic linear chain model to show the distributions of long (L) and short (S) Mn$\mathrm{O}$ bonds and $\mathrm{Mn}-\mathrm{Mn}$ distances. The net elongation is a weighted average of the different bond lengths and the net relative length change is much smaller than the variations of the $\mathrm{Mn}-\mathrm{O}$ bond lengths.

long and short Mn-Mn distances. Because the increase in length for the long bonds is larger than the decrease in length for the short bonds (including weighting factors) there is a net small change in the average $\mathrm{Mn}-\mathrm{Mn}$ distance as determined in thermal expansion or magnetostriction measurements. We illustrate this in Fig. 4. The top line shows the undistorted chain of Mn-O-Mn while the lower one shows the (exaggerated) distortions when some JT distortions are along the chain (short Mn-O bonds) and others are perpendicular to it (long $\mathrm{Mn}-\mathrm{O}$ bonds). The relative changes in the local distances $\mathrm{Mn}-\mathrm{O}$ and $\mathrm{Mn}-\mathrm{Mn}$ can be large, but the average relative change in the lattice constant, which is a weighted average of the long and short Mn-Mn distances is small.

\section{NEGATIVE THERMAL EXPANSION MATERIALS}

The third example we consider is the system $\mathrm{ZrW}_{2} \mathrm{O}_{8}$ which has a negative thermal expansion over a very wide temperature range $0.5 \mathrm{~K}$ to $1000 \mathrm{~K}[18]$. This material has an open crystal structure and it is composed of large corner sharing units - $\mathrm{WO}_{4}$ tetrahedra (two types $\mathrm{W}(1) \mathrm{O}_{4}$ and $\left.\mathrm{W}(2) \mathrm{O}_{4}\right)$ and $\mathrm{ZrO}_{6}$ octahedra. The $\mathrm{ZrO}_{6}$ octahedra form a FCC lattice (the octahedra are tilted) and are connected together via the $\mathrm{WO}_{4}$. However, one of the corners of each $\mathrm{WO}_{4}$ (oriented along a (111) direction) is not connected and leaves the $\mathrm{WO}_{4}$ partially unconstrained. In particular it can easily vibrate along the corresponding (111) axis. For this system the tetrahedra and octahedra units are considered to be rigid units; it is the correlated motions of these larger units that leads to the unusual thermal contraction properties. The resulting optical vibration modes are called Rigid Unit Modes (RUM) and have been used to describe the $\mathrm{ZrW}_{2} \mathrm{O}_{8}$ system[19]. However the explicit mode or sum of modes that leads to the thermal contraction has not been clear.

Using XAFS we have found an unusual behavior in the broadening of the pair distribution functions for some atom pairs while others such as W-O and $\mathrm{Zr}-\mathrm{O}$ exhibit typical behavior. In Fig. 5 we plot $\sigma^{2}$ for the W-O, W-Zr and $\mathrm{W}(1)-\mathrm{W}(2)$ neighbors. $\sigma_{W O}^{2}$ is essentially independent of $\mathrm{T}$, verifying that the $\mathrm{WO}_{4}$ tetrahedra are indeed very rigid. The weak temperature dependence indicates a large Debye (or Einstein) temperature of order $1000 \mathrm{~K}$. In contrast, $\sigma^{2}$ for the $\mathrm{W}(1)-\mathrm{W}(2)$ pair increases rapidly with $\mathrm{T}$, and a comparison of $\sigma_{W W}^{2}$ with the $\mathrm{U}^{2}$ parameters[20] for $\mathrm{W}$ indicate that the displacements of $\mathrm{W}(1)$ and $\mathrm{W}(2)$ are partially negatively correlated $(\phi \sim$ -0.4 at $300 \mathrm{~K}$ ). More surprising is the behavior of $\sigma^{2}$ for the $\mathrm{W}-\mathrm{Zr}$ atom pairs (W(1)-Zr and $\mathrm{W}(2)-\mathrm{Zr}$ added together). This linkage is also quite stiff, clearly much stiffer than the W(1)-W(2) 


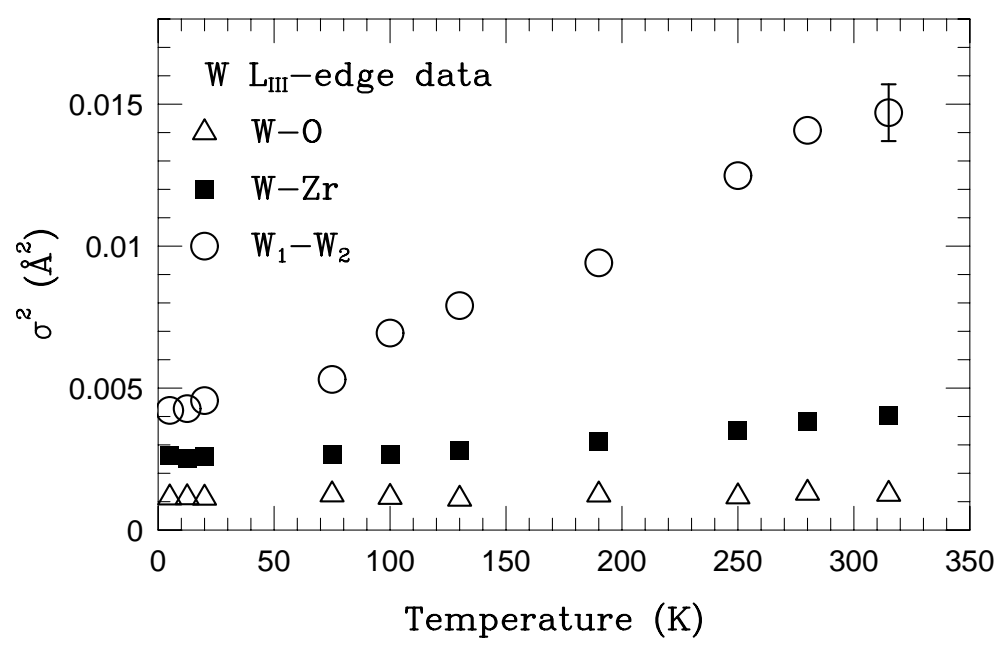

Figure 5: A plot of the broadening parameter $\sigma^{2}$, for $\mathrm{W}-\mathrm{O}, \mathrm{W}-\mathrm{Zr}$, and $\mathrm{W}(1)-\mathrm{W}(2)$ as a function of temperature. The very weak temperature dependence for $\mathrm{W}-\mathrm{O}$ indicates that this bond is very stiff - consistent with the assumption that $\mathrm{WO}_{4}$ is a rigid unit. $\sigma^{2}$ for $\mathrm{W}(1)-\mathrm{W}(2)$ increases rapidly with $\mathrm{T}$ and the large magnitude of $\sigma^{2}$ at high $\mathrm{T}$ indicates that the $\mathrm{W}(1)-\mathrm{W}(2)$ displacements are partially negatively correlated.

linkage even though the atom pair distances are nearly the same. This suggests that the rigid units that need to be considered are larger than just octahedra or tetrahedra. Fig. 6 shows one possible larger unit - an equilateral triangle of $\mathrm{Zr}$ surrounding a $\mathrm{W}$ atom (O atoms not shown). The XAFS data indicate that the $\mathrm{W}$ have large relative vibrations - if the $\mathrm{Zr}-\mathrm{W}$ linkage remains quite stiff, then motions of the $\mathrm{WO}_{4}$ tetrahedra along a (111) direction (perpendicular to the plane of the figure) will drive the motions of the $\mathrm{ZrO}_{6}$. In this figure, if the $\mathrm{W}$ moves out of the plane of the $\mathrm{Zr}$ atoms, the $\mathrm{Zr}$ atoms would have to move closer together to keep the $\mathrm{W}-\mathrm{Zr}$ distance fixed. Thus in this system, the correlated motions of large arrays of atoms appears to be crucial for understanding the macroscopic properties.

\section{CONCLUSIONS}

We have discussed the local atomic distortions in a number of more complex systems. In some materials, the local distortions are a superposition of displacements produced by two or more different mechanisms, as is the case for the quasi-cubic manganites which exhibit both colossal magnetoresistance (CMR) and charge ordering. For such systems it is useful to decompose the observed structural changes (broadening of the pair distribution function, thermal expansion, magnetostriction, etc.) into components associated with each mechanism; for the CMR systems, the two main mechanisms are thermally generated phonons and the motion of polarons. Once the net displacements are viewed as a sum of components, there is no need to consider unusual anharmonic behavior, at least for these systems, at temperatures below $300 \mathrm{~K}$.

We have also discussed correlations in the displacements of various pairs of neighboring atoms and given examples of both positive (in-phase) correlations and negative (out of phase, or 


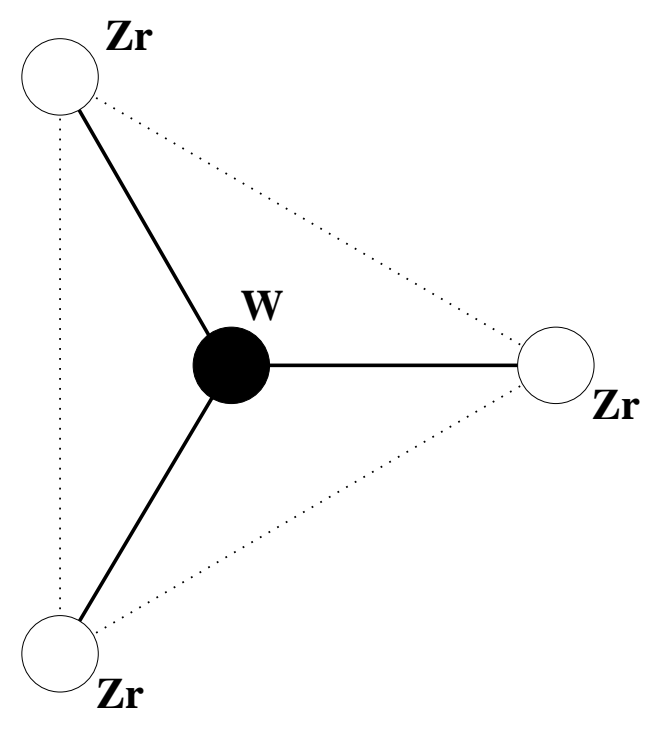

Figure 6: A simplified model of the unit formed of three $\mathrm{ZrO}_{6}$ octahedra and a $\mathrm{WO}_{4}$ tetrahedron. The XAFS data indicate that the $\mathrm{W}-\mathrm{Zr}$ linkage is stiff. Then if the $\mathrm{W}$ moves out of the page, the $\mathrm{Zr}$ atoms must move together.

anti-) correlations. In many materials the nearest neighbor atoms move together as a unit. This occurs both for systems for which the excited phonon modes are primarily acoustic and also in more complex crystals in which the structure can be viewed as a number of larger rigid units (octahedra, tetrahedra, etc.) connected together. This produces in XAFS the well-known, very narrow width for the nearest neighbor atom PDF.

Examples of negatively correlated displacements are less common but are found in some more complex structures. However, the effects of such correlations are less well understood. When present they lead to very large values of the broadening parameter $\sigma$.

Once rigid units are identified, their displacements and rotations need to be considered separately. For the negative thermal expansion materials the displacements of the nearest W-W pair are partially negatively correlated while the $\mathrm{W}-\mathrm{Zr}$ pair remains quite stiff. This suggests that a large unit composed of $3 \mathrm{ZrO}_{6}$ and a $\mathrm{WO}_{4}$ needs to be considered. Within this larger unit, displacements of the $\mathrm{W}$ will make the $\mathrm{Zr}$ atoms contract together.

The importance of correlations in the local atomic displacements is beginning to be recognized particularly for complex structures. However, the extent and nature of the rigidity of various units has not yet been explored in a wide range of materials and negatively correlated displacements have seldom been investigated.

\section{ACKNOWLEDGMENTS}

The experiments were performed at the Stanford Synchrotron Radiation Laboratory, which is operated by the U.S. Department of Energy, Division of Chemical Sciences, and by the NIH, Biomedical Resource Technology Program, Division of Research Resources. Some experiments were carried out on UC/National Laboratories PRT beam time. The work is supported in part by 
NSF grants DMR-97-05117 and DMR-00-71863, and by the Office of Basic Energy Sciences, Chemical Sciences Division of the U. S. Department of Energy, Contract no.

DE-AC03-76SF00098.

\section{References}

[1] B. K. Teo, EXAFS: Basic Principles and Data Analysis (Springer-Verlag, New York, 1986).

[2] C. H. Booth, F. Bridges, E. D. Bauer, G. G. Li, J. B. Boyce, T. Claeson, C. H. Chu, and Q. Xiong, Phys. Rev. B 52, R15745 (1995).

[3] C. H. Booth, F. Bridges, J. Boyce, T. Claeson, B. M. Lairson, R. Liang, and D. A. Bonn, Phys. Rev. B 54, 9542 (1996).

[4] A. P. Ramirez and G. R. Kowach, Phys. Rev. Lett. 80, 4903 (1998).

[5] C. Zener, Phys. Rev. 82, 403 (1951).

[6] P. W. Anderson and H. Hasegawa, Phys. Rev. 100, 675 (1955).

[7] P. G. de Gennes, Phys. Rev. 118, 141 (1960).

[8] C. H. Booth, F. Bridges, G. H. Kwei, J. M. Lawrence, A. L. Cornelius, and J. J. Neumeier, Phys. Rev. Lett. 80, 853 (1998).

[9] C. H. Booth, F. Bridges, G. H. Kwei, J. M. Lawrence, A. L. Cornelius, and J. J. Neumeier, Phys. Rev. B 57, 10440 (1998).

[10] G. Subías, J. García, M. G. Proietti, and J. Blasco, Phys. Rev. B 56, 8183 (1997).

[11] S. J. L. Billinge, R. G. DiFrancesco, G. H. Kwei, J. J. Neumeier, and J. D. Thompson, Phys. Rev. Lett. 77, 715 (1996).

[12] D. Cao, F. Bridges, D. C. Worledge, C. H. Booth, and T. Geballe, Phys. Rev. B 61, 11373 (2000).

[13] A. J. Millis, P. B. Littlewood, and B. I. Shraiman, Phys. Rev. Lett. 74, 5144 (1995).

[14] A. J. Millis, B. I. Shraiman, and R. Mueller, Phys. Rev. Lett. 77, 175 (1996).

[15] H. Röder, J. Zang, and A. R. Bishop, Phys. Rev. Lett. 76, 1356 (1996).

[16] D. Cao, F. Bridges, C. H. Booth, and J. J. Neumeier, Phys. Rev. B 62, 8954 (2000).

[17] F. Bridges, G. Brown, D. Cao, and M. Anderson, J. Synchrotron Rad. 8, 366 (2001).

[18] T. A. Mary, J. S. O. Evans., T. Vogt, and A. W. Sleight, Science 272, 90 (1996).

[19] A. K. A. Pryde, K. D. Hammonds, M. T. Dove, and V. H. etal, J. Physics: Condens. Matter 8, 10973 (1996). 
[20] J. D. Jorgensen, Z. Hu, S. Teslic, D. N. Argyriou, S. Short, J. S. O. Evans, and A. W. Sleight, Phys. Rev. B 59, 215 (1999). 